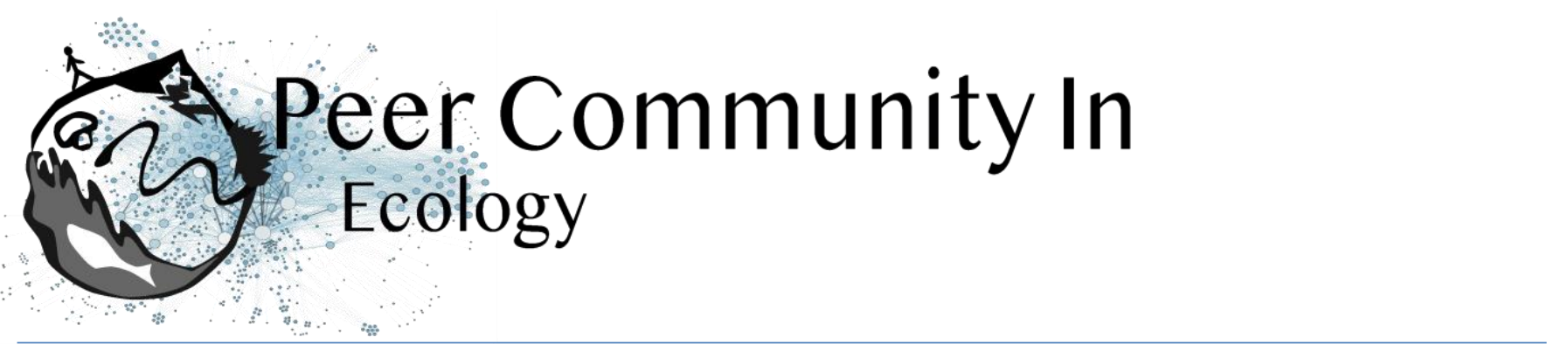

\title{
Stasis and the phenotypic gambit
}

\author{
Tom Van Dooren based on reviews by Jacob Johansson, Katja \\ Räsänen and 1 anonymous reviewer
}

\section{A recommendation of:}

Tim Coulson. Environmental perturbations and transitions between ecological and evolutionary equilibria: an eco-evolutionary feedback framework (2020), bioRxiv, 509067, ver. 4 peer-reviewed and recommended by Peer Community in Ecology. 10.1101/509067

\section{Open Access}

Submitted: 03 January 2019, Recommended: 14 June 2020

Cite this recommendation as:

Tom Van Dooren (2020) Stasis and the phenotypic gambit. Peer Community in Ecology,

100053. 10.24072/pci.ecology.100053

Published: 16 June 2020

Copyright: This work is licensed under the Creative Commons Attribution-NoDerivatives 4.0 International License. To view a copy of this license, visit http://creativecommons.org/licen ses/by-nd/4.0/
The preprint "Environmental perturbations and transitions between ecological and evolutionary equilibria: an eco-evolutionary feedback framework" by Coulson (2020) presents a general framework for evolutionary ecology, useful to interpret patterns of selection and evolutionary responses to environmental transitions. The paper is written in an accessible and intuitive manner. It reviews important concepts which are at the heart of evolutionary ecology. Together, they serve as a worldview which you can carry with you to interpret patterns in data or observations in nature. I very much appreciate it that Coulson (2020) presents his personal intuition laid bare, the framework he uses for his research and how several strong concepts from theoretical ecology fit in there. Overviews as presented in this paper are important to understand how we as researchers put the pieces together.

A main message of the paper is that resource detection and acquisition traits, broadly called "resource accrual traits" are at the core of evolutionary dynamics. These traits and the processes they are involved in often urge some degree of individual specialization. Not all traits are resource accrual traits all the time. Guppies are cited as an example, which have traits in high predation environments that make foraging easier for them, such as being less conspicuous to predators. In the absence of predators, these same traits might be neutral. Their colour pattern might then contribute much less to the odds of obtaining resources.

"Resource accrual" reminds me of discussions of resource holding potential (Parker 1974), which can be for example the capacity to remain on a bird feeder without being dislodged. However, the idea is much broader and aggression does not need to be important for the acquisition of resources. Evolutionary success is reserved for those steadily obtaining resources. This recalls the pessimization principle of Metz et al. (2008), which applies in a restricted set of situations and where the strategy which persists at the lowest resource levels systematically wins evolutionary contests. If this principle would apply universally, the world then inherently become the worst possible. Resources determine energy budgets and different life history strategies allocate these differently 
to maximize fitness. The fine grain of environments and the filtration by individual histories generate a lot of variation in outcomes. However, constraint-centered approaches (Kempes et al. 2019, Kooijman 2010) are mentioned but are not at the core of this preprint. Evolution is rather seen as dynamic programming optimization with interactions within and between species. Coulson thus extends life history studies such as for example Tonnabel et al. (2012) with eco-evolutionary feedbacks. Examples used are guppies, algae-rotifer interactions and others. Altogether, this makes for an optimistic paper pushing back the pessimization principle.

Populations are expected to spend most of the time in quasi-equilibrium states where the long run stochastic growth rate is close to zero for all genotypes, alleles or other chosen classes. In the preprint, attention is given to reproductive value calculus, another strong tool in evolutionary dynamics (Grafen 2006, Engen et al. 2009), which tells us how classes within a population contribute to population composition in the distant future. The expected asymptotic fitness of an individual is equated to its expected reproductive value, but this might require particular ways of calculating reproductive values (Coulson 2020). Life history strategies can also be described by per generation measures such as Ro (currently on everyone's radar due to the coronavirus pandemic), generation time etc. Here I might disagree because I believe that this focus in per generation measures can lead to an incomplete characterization of plastic and other strategies involved in strategies such as bet-hedging. A property at quasi-equilibrium states is precise enough to serve as a null hypothesis which can be falsified: all types must in the long run leave equal numbers of descendants. If there is any property in evolutionary ecology which is useful it is this one and it rightfully merits attention.

However, at quasi-equilibrium states, directional selection has been observed, often without the expected evolutionary response. The preprint aims to explain this and puts forward the presence of non-additive gene action as a mechanism. I don't believe that it is the absence of clonal inheritance which matters very much in itself (Van Dooren 2006) unless genes with major effect are present in protected polymorphisms. The preprint remains a bit unclear on how additive gene action is broken, and here I add from the sphere in which I operate. Non-additive gene action can be linked to non-linear genotype-phenotype maps (Van Dooren 2000, Gilchrist and Nijhout 2001) and if these maps are non-linear enough to create constraints on phenotype determination, by means of maximum or minimum phenotypes which cannot be surpassed for any combination of the underlying traits, then they create additional evolutionary quasi-equilibrium states, with directional selection on a phenotype such as body size. I believe Coulson hints at this option (Coulson et al. 2006), but also at a different one: if body size is mostly determined by variation in resource accrual traits, then the resource accrual traits can be under stabilizing selection while body size is not. This requires that all resource accrual traits affect other phenotypic or demographic properties next to body size. In both cases, microevolutionary outcomes cannot be inferred from inspecting body sizes alone, either resource accrual traits need to be included explicitly, or non-linearities, or both when the map between resource accrual and body size is non-linear (Van Dooren 2000).

The discussion of the phenotypic gambit (Grafen 1984) leads to another long-standing issue in evolutionary biology. Can predictions of adaptation be made by inspecting and modelling individual phenotypes alone? I agree that with strongly non-linear genotype-phenotype maps they cannot and for multivariate sets of traits, genetic and phenotypic correlations can be very different (Hadfield et al. 2007). However, has the phenotypic gambit ever claimed to be valid globally or should it rather be used locally for relatively small amounts of variation? Grafen (1984) already contained caveats which are repeated here. As a first approximation, additivity might produce quite correct predictions and thus make the gambit operational in many instances. When important individual traits are omitted, it may just be misspecified. I am interested to see cases where the framework Coulson (2020) proposes is used for very large numbers of phenotypic and genotypic attributes. In the end, these highly dimensional trait distributions might basically collapse to a few major axes of variation due to constraints on resource accrual.

I highly recommend reading this preprint and I am looking forward to the discussion it will generate.

\section{References}


[1] Coulson, T. (2020) Environmental perturbations and transitions between ecological and evolutionary equilibria: an eco-evolutionary feedback framework. bioRxiv, 509067, ver. 4 peer-reviewed and $\begin{array}{llll}\text { recommended by } \quad \mathrm{PCl} \text { Ecology. } & \text { doi: 10.1101/509067 }\end{array}$ [2] Coulson, T., Benton, T. G., Lundberg, P., Dall, S. R. X., and Kendall, B. E. (2006). Putting evolutionary biology back in the ecological theatre: a demographic framework mapping genes to communities. Evolutionary Ecology Research, 8(7), 1155-1171. [3] Engen, S., Lande, R., Sæther, B. E. and Dobson, F. S. (2009) Reproductive value and the stochastic demography of age-structured populations. The American Naturalist 174: 795-804. doi: 10.1086/647930 [4] Gilchrist, M. A. and Nijhout, H. F. (2001). Nonlinear developmental processes as sources of dominance. Genetics, 159(1),

423-432.

[5] Grafen, A. (1984) Natural selection, kin selection and group selection. In: Behavioural Ecology: An Evolutionary Approach,2nd edn (JR Krebs \& NB Davies eds), pp. 62-84. Blackwell Scientific, Oxford. [6] Grafen, A. (2006). A theory of Fisher's reproductive value. Journal of mathematical biology, 53(1), 15-60. doi: 10.1007/s00285-006-0376-4

[7] Hadfield, J. D., Nutall, A., Osorio, D. and Owens, I. P. F. (2007). Testing the phenotypic gambit: phenotypic, genetic and environmental correlations of colour. Journal of evolutionary biology, 20(2), 549-557. doi: 10.1111/j.1420-9101.2006.01262.x

[8] Kempes, C. P., West, G. B., and Koehl, M. (2019). The scales that limit: the physical boundaries of evolution. Frontiers in Ecology and Evolution, 7, 242. doi: 10.3389/fevo.2019.00242 [9] Kooijman, S. A. L. M. (2010) Dynamic Energy Budget theory for metabolic organisation. University Press, third edition. [10] Metz, J. A. J., Mylius, S.D. and Diekman, O. (2008) When does evolution optimize?. Evolutionary Ecology Research 10:

629-654.

[11] Parker, G. A. (1974). Assessment strategy and the evolution of fighting behaviour. Journal of theoretical Biology, 47(1), 223-243. doi: 10.1016/0022-5193(74)90111-8 [12] Tonnabel, J., Van Dooren, T. J. M., Midgley, J., Haccou, P., Mignot, A., Ronce, O., and Olivieri, I. (2012). Optimal resource allocation in a serotinous non-resprouting plant species under different fire regimes. Journal of Ecology, 100(6), 1464-1474. doi: 10.1111/j.1365-2745.2012.02023.x [13] Van Dooren, T. J. M. (2000). The evolutionary dynamics of direct phenotypic overdominance: emergence possible, loss probable. Evolution, 54(6), 1899-1914. doi: 10.1111/j.0014-3820.2000.tb01236.x [14] Van Dooren, T. J. M. (2006). Protected polymorphism and evolutionary stability in pleiotropic models with trait-specific dominance. Evolution, 60(10), 1991-2003. doi: 10.1111/j.0014-3820.2006.tb01837.x

\section{Revision round \#2}

2020-04-16

This manuscript by Coulson previously called "Causes Of Death And Failures To Reproduce, Limiting Resources, Ecological Dynamics, And Selection: How To Evolve A Low Predation Guppy, And Cause A Trophic Cascade", presents a thorough revision which addresses most of the previous comments by two reviewers and myself adequately. The two reviewers have a number of remaining remarks, which in my view can almost all be addressed with some rewording or the addition of references.

In my view, this manuscript in a way provides a generalization of the concept of "resource holding power" to a broader eco-evolutionary context. On line 555 the manuscript cites a redefinition of fitness in terms of energy, but there are usually several resources required to complete a life cycle and as L561 states, there can be several primary causes of death and failure to breed. I would appreciate it if the manuscript could give a bit more attention to situations where there are several primary causes or limiting resources, or stress the conditionality of an argumentation on there being a single primary cause or limiting resource when that is the case. 
Reviewer comments on "Environmental perturbations and transitions between ecological and evolutionary equilibria: an eco-evolutionary feedback framework"

This comprehensive, revised manuscript provides a conceptual and mathematical framework for understanding equilibria of ecological and evolutionary processes. The manuscript aims to link processes regulating population growth and community dynamics with evolution of species interactions and resource accrual \& life-history traits. Overall, I find the manuscript very useful and interesting - and it was an enjoyable read for the majority of the individual sections. Even for a "modelling dummy" like myself, it really managed to explain the processes and models in a comprehensive and intuitive way.

At the same time, there are some aspects that I think still could be improved to make it an easier read. Particularly is it is a very long $\mathrm{ms}$ and the topic complex. The core comments relate to better addressing \& acknowledging the pertinent literature at places, clarify some conceptual points and to further structure it (e.g. by add subheadings in few places) to facilitate reading also for non-experts. The figures are clear and useful.

Below I make an overview (to guide also myself through the complexity of the text), some major and some more specific (including many minor) suggestions. I hope these are helpful and aid in further improving the manuscript.

\section{Overview}

As the manuscript is long and covers many core concepts and processes, I find it useful to provide an overview of the sections as follows. (I would recommend the author also to consider such overview before going into the actual text to give the reader a frame to follow). It starts from introducing the classic empirical case of the high and low predation adapted Trinidadian guppies, and asking what determines ecological and evolutionary (quasi)-equilibrium in either a high or low predation state. It then introduces the paradox of stasis and the paradox of maintenance of genetic variance and touches upon species co-existence theory and different levels of biological organization. The focus is on several places on resource accrual traits (i.e. traits that influence ability to detect and acquire resources). After the introduction section, the manuscript covers (with some notes):

1) A mathematical break down of demographic population models in the context of Eco and Evo equilibria (which is very nicely done). 2) Conditions that are required for stasis in Eco and Evo processes. 3) Potential solutions to the paradox of stasis in the context of quantitative genetics - This is a rather lengthy and complex section (including various processes from life-history theory to stabilizing selection and frequency dependent selection) - but informative (see specific comments below). 4) Focus on body size as a major resource accrual trait and how body size links to population growth rate and its dependence on environmental sources of variance 5) Population models, focusing on structural and individual based models particularly IPMs • suggesting that models based on demographic, developmental and inheritance functions can aid in understanding eco-evolutionary dynamics (broadly speaking). It explains the IPMs in more detail in this context. 6) Exploration on how systems can transition between different equilibria states - as a consequence of abrupt environmental change. 7) The ms then ends with speculating on processes that may have been driving the evolution of the emu (to a large bodied, flightless bird via resource and predation mediated selection) and returns briefly to the Trinidadian guppies and trophic cascades.

Major comments:

- In several locations, I still feel there is a jump in the logic - I indicate below (by JMP) where I noticed it. Perhaps it will help to make some simple adjustments with concrete statements that link across 


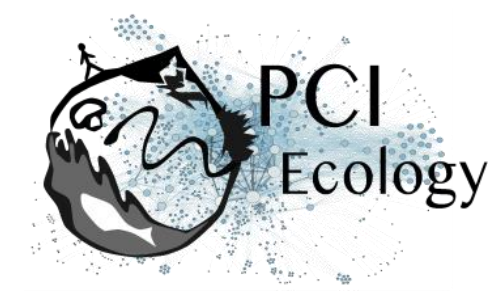

paragraphs in these situations. For this case, using some subheadings in select long paragraphs where there is a topic shift might most easily solve the issue.

- In several cases also core references should be given - I indicate those below by REF.

- Where wording should be corrected or checked, I use RWD for "reword"

I still think the section on Emma-Steve is too speculative. No references are given what the current hypotheses are for the evolution of body size and flightless ness in ratites - or if there is any evidence that would support body size as an resource accrual trait (L895-896). I am by no means either a phylogeneticist or phylogeographicist - but it seems to me (by a brief check of recently published literature, such as Faux \& Field 2017, Yonazewa et al. 2017; Thomson et al. 2018) that there is much discussion about what drove the evolution of flightless ness and large body size of ratites, and body size evolution of emu's. To write this section without that context in mind, is not in my opinion useful - neither really appropriate. So either the context of open questions - and to what extent there is evidence for resources and/or predation to have influenced their evolution - should be widened (briefly), or then this section should be left out. As fun as it is and as cute - I am sure - Emma-Steve was, the current treatment is a bit "lose".

Detailed comments

P3: I still struggle a bit to get at the core of the paper when we start by a general description of the Guppy system. But this may be a matter of style.

L45-46 would more logically be moved after L33-34. Core general REFs should be provided at L33-34 already.

L60: REF needed

L68-69: There is something odd with the wording here. Perhaps RWD to "The genetic, phenotypic trait, xxxx and sex structure of all populations..." ?

L71: RWD "This means that there may be..."

L74 - I do not follow the logic here from L71-73 to L74 about species going extinct.

L84 - JMP in logic from evolutionary equilibrium to definition of phenotypic traits.

L88 - RWD to “...under directional selection, and heritable, but do not evolve..."

L90 - is this full sentence needed ?

L99-100 - I am missing some of the logic here. If phenotypic traits are at optimum, presumably there would be no selection and additive genetic variation would be maintained? (Else I see the "no paradox" in additive genetic variation being maintained in presence of selection - be it directional or stabilizing).

\section{L117-118: JMP across paragraphs}

L120 - Here I assume you refer to the "selective environment" within which an individual performs. What about the developmental environment which may influence phenotypic variation in resource accrual traits? I see this comes later in the text, but might be useful here to clarify which aspect of the environment you refer to.

\section{L125: Add REF}

L131: Add REF. The role of transgenerational plasticity (TGP) is not addressed - although you refer to nongenetic inheritance. Would TGP possibly affect the potential for ecological or evolutionary stasis (for example)? I don't see a need to dwell in this in any length - but given theoretical and empirical work indicating that TGPs can drive/influence population dynamics - it may be useful to briefly refer to such work.

L142: State (see REF above) or provide couple of REFs as a reminder to the reader.

L147: RWD. Should this be "...evolution also optimizes .." ? 


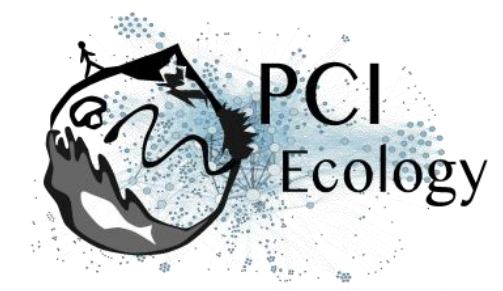

L155 - RWD. Something missing in the logic/wording relating to the limiting factor.

L157 - This rapid change in population size and selective regimes would presumably require rapid environmental change also. Clarify.

L188 - RWD to .."there may be 23 individuals.."

L211 - What about environmental effects on gene expression - which would possibly influence genetic inheritance?

L234 - Clarify how genotype-by-environment interactions and non-genetic inheritance are dealt with in these models. (And if they are).

L243 - JMP in logic between the two sentences.

L265-266 - A subheading would be helpful between these two paragraphs.

L283-284 - Provide REF.

L301- JMP in logic. Add a lead sentence between paragraphs.

L321-326 paragraphs seem to me somewhat repetitive from earlier in the $\mathrm{ms}$. If so, consider shortening given the extensive length of the manuscript.

L333-335 - Should this not include "in a density dependent manner" ? For example, when the effects of predation, herbivory or resource availability affect population density and therefore per capita population growth rate?

L356 - RWD to... "workS by..."

L406-407 - I think moving this first - and thereby tying the sentences about rotiger-algae system better together would make the reasoning easier to follow. This would seem to call for key REFs for ecoevolutionary dynamics literature (would benefit the broader audience).

L408 - RWD to "...rotifer population laggING a half cycle.."

L409-418 - Calls for a REF. I seem to recall that the mechanisms related to clumping vs soloists in the ecoevolutionary dynamics were considered in a separate paper by Becks et al. 2010 (Ecol. Lett).

L423-428 -This section is repetitive to above. Consider shortening.

L435- A subheading may be useful here given a JMP in the content

L437-438 - RWD to "......observed, for example, in the Trinidadian freshwater streams (See Introduction)..

L444 - I would state " determined by the association between phenotypic trait values and fitness (i.e. survival and reproduction)".

L448 - how is the environmentally determined variance (= phenotypic plasticity) considered in this genotypephenotype map?

L449. RWD to “...trait values and fitness.".

L452 - RWD to "...where the highest fitness is observed at..."

L478 - REF to the concept of individual specialization here (papers by Bolnick \& co)

L483 - a reminder of REFs to the storage effect would be useful (given the length of the paper finding the appropriate location for what the "storage effect" is not so quick).

L487-488 - RWD perhaps to "When these processes are operating, equilibria can be achieved, whereby each species, and ....has a long-run stochastic growth rate of zero." 
L490 - RWD to “includING"

L495 - Provide a REF to phenotypic gambit as a reminder for non-experts in the field. Though I am not sure if we need to evoke the phenotypic gambit in this sentence - given that quantitative genetic partitioning in its self does not yet imply that we can use estimates of VA as predictors of evolutionary dynamics. So the gambit part of the usage, perhaps would rather be brought up a bit later when aiming to infer evolutionary processes.

L503-507. Refer to those "other" - sometimes substantial - sources of phenotypic variance more explicitly than just saying "can be partitioned into various other"... This over simplification of assuming VP = VA + VE should be better justified (e.g. from the perspective that it is the narrow sense heritability that is assumed to matter for evolutionary responses - although this is not strictly true).

L510 - RWD to "...and the traits are heritable..."

L511-515 - The jump to body size comes a bit abruptly. Perhaps move L528 lead sentence here and to draw attention to why size related traits are of interest. We should also keep in mind that although size related traits are heritable (in terms of harbouring additive genetic variation), they also are classic examples for traits harbouring substantial phenotypic plasticity.

L516-517 - I assume it is not the individuals that have alpha = 0, but genotypes or populations. RWD sentence for clarity.

L528 - See comment for L511-515.

L533 - and also why not individual size in all populations ? (if taking a micro- to macroevolution process thinking).

L571 - A REF would be useful here.

L571-572 - A subheading would be useful given the lengthy part of this solution to the paradox section

L583-587 - Seems somewhat repetitive from the early parts of the manuscript. Perhaps simplify and refer to "as above".

L598-601 seems to come as a logic JMP and could be better tied to the text before and after.

L620-622 - RWD. This sentence of body size not evolving and its link to resource accrual trait and optimal values is unclear. Something like "When body size is heritable and under directional selection, there will be no evolution of larger body size because the mean trait value of this resource accrual trait is at an optimum when the population has achieved an evolutionary equilibrium". (If I now understand this correctly).

L650-652 - something missing in logic. You mean "...such that body size $Z$ is not $A+E$, but rather $A+E / A$ ". ?

L655-657. I find this statement not to be well framed to the theory - Modern quantitative genetic approaches and attempts to predict evolutionary responses are increasingly indicating that predictions based on additive genetic variance and h2 are often not accurate - for example due to the various alternative sources of phenotypic variance and the relative contribution of environmental variance differing in different environments (e.g. populations) and traits. Perhaps I am missing the point here but it would be useful to REF to recent other papers that have aimed to address the issue that $r=h 2 * s$ does not often predict accurately evolutionary changes in traits.

L656-657 - A logic JMP here between previous and invoking body size. Can this last sentence be left out or tied to the start of the next paragraph (L659 onwards) ?

L662-664 - I don't follow the logic here, RWD needed. You mean body size is a consequence of how aggressive individuals are? If so, you mean their developmental trajectories were influenced by behavioural aggression ? Or there is in fact a genetic correlation between body size and behavioural aggression? 


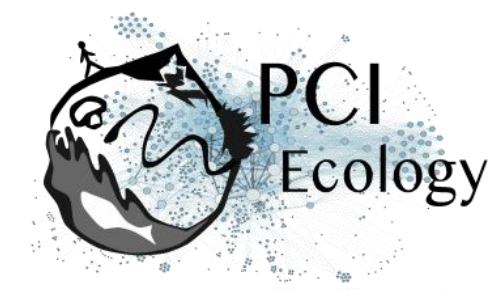

L677 - RWD to "...is THAT they may be able to..."

L687 - core original REFs for Bergmann's rule should be provided

L690-691 - I don't follow the reasoning in this sentence. RWD to "In cases where body size...harbours heritable (additive genetic) variation .." - I don't understand the latter part of the sentence - what do you mean "as predated by evolution" ?

L690-700 onwards - I am not sure this predictable evolutionary responses in artificial environments is needed in this length. I think a shorter statement would suffice.

L704-L705 - I thought above also was about the paradox of stasis? Rather perhaps RWD to "Next I will show how population models can be constructed to address the paradox of stasis".

L716 - RWD? You mean to say that models can be structured (e.g. by size or age class) or individual-based ?

L735 - RWD to "...need to be explicitly combined.."

L753-755 \& L766-769 are partially redundant. Combine. (I suggest removing L753-755).

L775-777 - What about the role of transgenerational plasticity in these transition functions? They may have rather strong impacts on how environmental effects of parental generations influence offspring phenotype and performance in the offspring environment (and hence natural selection).

L804 - RWD to “...would be TO construct..."

L806-808 - Just a note: environmental effects on body size can be other than resource related (such as physiological stress that may reduce body size, and organisms due to cellular processes may not be able to compensate even when resources per se would be abundant).

L820 - which "this" trait?

L830 - the sudden shifts that organisms experience as abiotic change due to volcanic eruption is not the volcanic eruption per se, but rather changes that occur as consequence (e.g. elevated toxicants in the air). Perhaps chemical spill would be a more appropriate direct abrupt abiotic example?

L838-840 - I find the argumentation here about role of plasticity in reducing rate of evolution possibly unclear to the non-experts. I suggest stating this more explicitly and referring to pertinent literature. Also, although such novel selection may initially result in changes in trait mean, this may change when plasticity is costly or insufficient - whereby there would be selection at the genetic level when plasticity is not available or sufficient. (NB. I would also consider transgenerational plasticity as a modifier of phenotypic change and responses to selection).

L848 - The argument about considering key stone species is valid, but comes here with a logic JMP. Also RWD to "If a change in A limiting factor, population size, and selection pressure.

L863 onwards - See my comment (above) about the need to address the open questions in ratite evolution rather than just stating a speculative hypothesis without known context in to the discussion.

L863 - Change heading to reflect order of text (emu, guppy, innovation)

L870 - you mean individuals ? (e.g. adaptive traits via plasticity and/or a genetic basis)

L901-902 - provide REFs for these arguments

L921 - provide some of the core REFs for role of evolutionary innovations as a bridge between micro- and macroevolution

L925-940 - this section is rather long and could be shortened to the key points, esp given the ms is already very long 
Dear editors,

I have reviewed the manuscript "Environmental perturbations and transitions between ecological and evolutionary equilibria: an eco-evolutionary feedback framework" which is a revised version of a previous manuscript submitted to $\mathrm{PCl}$.

The manuscript has been thoroughly revised and reworked and I am convinced it would be a valuable contribution to the field of evolutionary ecology and of interest for empiricists and theorists alike. The manuscript provides an interesting overview of how evolutionary and ecological stationarity may arise and be characterized. Just like the previous version, the manuscript is very well written and uses an efficient combination of theory and illustrative empirical examples to guide the reader through the many dynamical effects of biotic perturbations occurring at different levels, from birth and death processes and demography to population dynamics and evolutionary outcomes. Finally, the ms puts forward an interesting and novel framework to study ecological and evolutionary processes which combines integral projection models (addressing demographic dynamics and individual development) and a type of quantitative genetic model (I think you may say). The description of the model has been much improved in this version by more clearly describing the role of the transition and inheritance functions, resource accrual traits, a generic equation clarifying the model for theorists (L746) and new and much more instructive figures (figs. 3-5). Hence it should now be clearer to readers how this type of model may be constructed. Previous models/framework with a similar scope have also now been cited making it easier for readers to compare with previous work and related literature. It seems to me that this type of model could have a large appeal to empiricists since both population structure (e.g. size structure) and genetic structure (e.g. trait variance) structure in the model are described as continuous functions which can be related to data obtained in the field or in experiments.

I only have a few comments, which I hope can be useful for a final revision. They are a bit tricky to explain and I am sorry if they are a bit lengthy but I am convinced most of them can be addressed by adjusting the wordings and explanations in this review/perspective piece.

1. According to the abstract energy budgets are integrated into the framework. Then the dynamic energy budget model (DEB) by Kooijman is cited and energy/energy budgets are discussed intensively in the beginning and middle of the MS. However energy budgets seems to be absent in the section where the model framework is outlined. I suspect energy budget are an integrated part of the demographic or resource accrual function but it would not hurt to remind the reader in the model section. The model is outlined on approx. L739-827 but last mention of "energy" is before that on L699.

2. L249 "when genotypes are additive, genotypes within offspring cohorts are expected to have identical reproductive values (Sæther and Engen 2015).". This requires more explanation. I admit I have not consulted the paper by S\&E 2015, but I wonder if this is generally true under evolutionary stationarity in a stochastic world. Specifically I am thinking of mutation-selection balance which is an important characteristic of evolutionary stationarity. Under mutation-selection balance, mutants (i.e. genotypes) with fitness/reproductive value below 1 coexist with genotypes alongside those with the optimal phenotype (with $\mathrm{RO}=1$ or in fact ever so slightly above 1 so that the whole population including maladaptive types has mean RO=1). Without having thought too much about it, I can imagine the statement on L249 is true in a deterministic world but I doubt it is true when accounting for mutation-selection balance where necessarily some genotypes in the offspring cohorts might be maladaptive mutants. One may perhaps assume this is a very small effect if mutants are rare etc, but frequency and density dependence can generate very flat fitness landscapes (since species evolve towards any resource peaks and thereby press them down) so at evolutionary equilibrium mutants close but not on the optimum phenotype may have only a small fitness disadvantage and take very long time to weed out. As an example, I did some individual-based population genetic simulations 
under frequency dependent selection in Johansson, J. (2008). Evolution, 62: 421-435 where the population had a considerable standing genetic variation at ESS with three species due to mutationselection balance in a fitness landscape evolution had flattened out in this way (note I don't at all propose that paper should be cited here - it is not about mutation-selection balance and I am sure it is well covered elsewhere). Anyway, my point is that it is not intuitively clear that all offspring have the same reproductive value at an evolutionary stationary state, and if so rewording is needed at L249 and several other places in the MS where this line of thought appears (e.g. L289, 298, 491).

3. Following up on the point 2, I personally wonder if mutation-selection balance can generate a quite large standing genetic variation especially if you consider genetic variation in many loci, where alleles at each loci has small effects on fitness. We could then have many just slightly maladaptive genotypes that would take ages to weed out.

4. L13,L19,L20, L147, L307 and more places. The MS talks about optimization of traits, but whenever you have ecological feedback loops via trait evolution, trait optimization becomes problematic. If a predator trait affect the availability of different prey types, for example, the optimum trait would depend on the current trait values of the predators. Assume for example a novel environment with a lot of large prey and a few small prey. The optimum appears to be to eat large prey. But if all predators eat large prey, large prey would decrease in number and then it might be optimal to eat small prey. So we quickly get game-theory-like situations when ecological feedbacks are operating and game-theory has shown many examples where apparently sub-optimal strategies are viable at evolutionary equilibria (this is by the way of course a main thread in adaptive dynamics and has less to do with clonal inheritance). There may be specific instances where trait optimization is a valid principle, for example the vision trait discussed on. But more generally, I suggest some caution the word optimization being used with caution. There is a lot of tradition in R0 maximization, but this principle may only be valid in certain circumstances when you have ecological feedbacks, e.g. when population size is constant and regulated by a simple density dependence such as sibling competition. See e.g. Kozlowski Measuring fitness in life-history studies. Trends in Ecology \& Evolution 8:84-85 and Metz, J. A. J., Mylius, S. D., \& Diekmann, O. (2008). When does evolution optimize? Evolutionary Ecology Research, 10(5), 629-654 who argues it is very rare for R0maximization to be a justifiable principle.

5. L307. R0-optimization is extensively used in life history theory but not (or at least very rarely) in adaptive dynamics by Metz 1992, 1995 so this sentence needs rewording.

6. L309: "Adaptive dynamics and game theory consequently rely on the assumption of clonal inheritance." This statement is made without references, and not discussed before so they come a bit abruptly. It is also problematic because both these frameworks are quite diverse with many model versions. Game theory is a big area so there may be some games for sexually reproducing organisms out there somewhere and for adaptive dynamics, you perhaps intend to refer to the adaptive dynamics version by Metz and others, where you have the so called canonical equation of adaptive dynamics (Dieckmann and Law 1996) which is defined for asexual inheritance as this is easier (yet not easy...) to derive mechanistically, but in this broader school of thought you also find models with sexual inheritance, and as I pointed out in my previous review adaptive dynamics equations have been derived for sexual inheritance. Also, in an earlier branch of adaptive dynamics inheritance is modelled using quantitative genetics which in turn is often used to model sexual populations (see e.g. Vincent et al "Evolution via strategy dynamics." Theoretical Population Biology 44.2 (1993): 149-176.). I think it is easier to skip labels and simply refer to "approaches assuming clonal inheritance" and cite such papers.

7. L464. Two species with the same "optimal" fitness may appear able to coexist, but I am not sure it is generally true. Identical fitness rather leads to drift in the two populations and if you have simple density dependence (e.g. $n 1+n 2$ is constant) then in the long run there would be stochastic extinction of one of them just due to demographic stochasticity. A much stronger argument for 


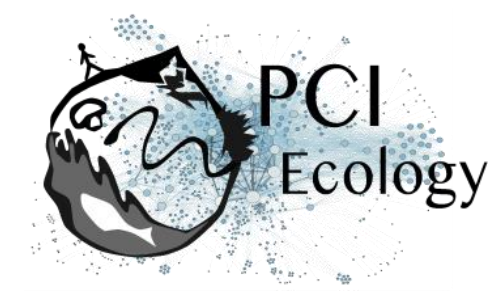

coexistence is negative frequency-dependent selection so that the rare genotype bounces back to equilibrium when rare.

8. L823. Here I wonder whether the model assumes there can only be a single size at maturation that maximizes fitness given this trade-off, or whether the model would be able to account for coexistence of small and large phenotypes, such as fighters and sneakers which differ in size at maturation yet are able to coexist on game-theoretical grounds (the hawk and dove game).

9. Fig. 4. Here all curves are smooth. But given e.g. interannual variability of the availability of different resources due to changing demographies of several populations I would expect the population size distributions and trait distributions to become irregular and deviate from smooth normal distributions etc. Or are there some simplifying assumptions here I have missed, for example about population sizes being very large?

Extremely minor comments:

1. Equation numbers needed on L668, L746

2. A couple of typos: L147 optimally->optimize L490 included-> including L907 specie->+s

Signed by Jacob Johansson

Author's reply:

Download author's reply (PDF file)

\section{Revision round \#1}

2019-02-25

Coulson proposes a framework to investigate what happens when a change in community composition alters the dominant cause of death (or of failure to reproduce) by changing the limiting resource. This is a topical issue. Immediately, it bears relevance to studies of context-dependent specialization of species, of potential effects of ecosystems going trough bifurcations (tipping points), although neither is treated. The manuscript is accessible and reads well. However it is for a large part a very lengthy description of required components of any realistic eco-evolutionary model that would be able to produce predictions for a particular system. These components are rather cursorily covered, none of the equations proposed are linked into a real model. Two reviewers and myself believe that this manuscript presents a relevant topic and that it is potentially worth recommending. However, a number of issues have to be addressed before we can make such a recommendation on behalf of $\mathrm{PCl}$ Ecology. Next to the points raised by the reviewers, I want to point out the following:

The guppy example leads us very easily into the issues addressed and I appreciate it very much. However, I believe the manuscript currently has too much focus on removing predators (or consumers, for plants), i.e., on community changes on a different trophic level than a focal species (the guppy). In another relevant scenario, coexisting competitors might each specialize on a different resource, becoming limiting for each of them. A removal of one competitor could then alter the eco-evolutionary feedback drastically.

Here is my most important remark: the manuscript lacks any definition of what a limiting resource is or of a key factor limiting population growth, and how one should define and determine "the dominant cause of death" or a factor controlling reproduction. This needs to be added. When these characteristics are not defined, they cannot be used to generate or assess predictions. I believe the author refers to a method like $\lambda$ contribution analysis, but this is not made explicit, so we can't assess whether that approach is entirely defensible for the intended purpose. 
Recently, a special issue has appeared in Functional Ecology on eco-evolutionary dynamics https://besjournals.onlinelibrary.wiley.com/toc/13652435/2019/33/1 with much attention for community effects of feedback loops in models and empirical systems. Coulson presents a mix of results on agestructured models, of modelling population and evolutionary dynamics in fluctuating environments, mostly using IPM's. Often the presentation does not do justice to the true origin of a concept. For example evolutionary suicide is not due to Rankin and Lopez-Sepulcre, but to Ferriere, Gyllenberg and Parvinen. Several modelling frameworks are just omitted (physiologically structured population modelling, epidemiological modelling using ODE's). I believe it is undoable to treat them all well. On the other hand, the bias towards IPM's has no obvious merit either. I would therefore prefer that the description of all model ingredients is drastically shortened to bring the focus back on the main issue: predict what happens when a sudden species change in an assemblage or community alters the eco-evolutionary feedback drastically.

The manuscript stays a bit unclear on what the timescale is where limiting resources will usually change. Is adaptation really relevant if species composition changes often? How often does a switch in key-factor occur? What with organisms that have a metamorphosis?

The manuscript treats the specialization of traits to environmental conditions. However, there is no mention of trade-offs. They need to be discussed, the focus is too much on single traits now (such as body size).

I believe the statement on p. 35 that no model combines all ingredients of Figure 5 is an overstatement. Please consult for example the models by Gavrilets and coworkers in the group of papers called "CASE STUDIES AND MATHEMATICAL MODELS OF ECOLOGICAL SPECIATION". I believe the link between the issue treated here and such models is strong and insufficiently treated. Consider Rundle and Schluter (2004. Natural selection and ecological speciation in sticklebacks), box 9.5: They use results of eco-evolutionary modelling to predict scenarios where speciation does not occur, but repeated invasions instead. The same trick can be applied for species removals. Hence I believe much of the framework needed exists and has been used to predict effects of changes in community composition, with results maybe needing a different interpretation in view of the questions asked here.

The Emu example: I would use it to propose colonization and evolution on islands as a general scenario where changes in limiting factor are relevant. For an overview of patterns of selection on small and large body size, please consult Blanckenhorn, W. U. (2000). The evolution of body size: what keeps organisms small?. The Quarterly review of biology, 75(4), 385-407.

Best regards, Tom Van Dooren

Preprint DOI: doi: https://doi.org/10.1101/509067

\section{Reviewed by Jacob Johansson, 2019-02-15 21:21}

I have read the manuscript "Causes of death and failures to reproduce, limiting resources, ecological dynamics, and selection: how to evolve a low predation guppy, and cause a trophic cascade" by Tim Coulson.

\section{OVERALL COMMENTS}

This manuscript is about the ecological and evolutionary consequences of removing a species from, or adding a species to, an interactive community. In particular the manuscript focuses on limiting resources and the possibility that adding or removing species might affect which resource becomes the most limited resource. As an illustrating example the manuscript starts out discussing how in the case of guppy communities, the presence of a predator could imply change the dominating selection pressures from being mainly determined by competition for food to being mainly determined by competition for predator-free space.

The manuscript is very well written and uses an efficient combination of theory and illustrative empirical examples to guide the reader through the many dynamical effects of biotic perturbations occurring at different levels, from birth and death processes and demography to population dynamics and evolutionary 
outcomes. Further the manuscript discusses how processes occurring at different levels may interact with each other and cause more or less surprising feedbacks. The paper culminates in the proposal of a modelling framework which integrates these multi-level dynamics.

I suppose the manuscript is intended as a forward-looking review or perspective article with the main aim of pointing out new research areas which becomes possible to study with an integrative approach to study ecoevolutionary responses. In its current form I think the manuscript provides many interesting thoughts, but I also think it could be improved if it would be linked more deeply to previous theory and modelling of ecoevolutionary dynamics in interacting communities.

Firstly, several papers not cited here have been devoted to the study of ecological and evolutionary consequences of species removal. These includes models which are simpler than the framework proposed here, e.g. Lotka-Volterra community models and adaptive dynamics (with much less genetic detail for example). I will provide some examples below. For this reason I think the manuscript would benefit from (A) outlining more precisely which aspects of species-removal responses require further study, and back this up with references and $(B)$ provide more precise arguments why the study of these phenomena require the use of the rather complex model framework proposed here. I am thinking of Occam or perhaps Einsten (?):"Everything should be made as simple as possible, but not simpler". An advantage of simpler models is that they are more transparent and often lend themselves to analytical treatments.

Secondly, and following up on point (B) above, there already exists some model frameworks (e.g. eco-genetic models, see below). which have a similar scope as the one proposed here. Thus, I think it is questionable if we actually need a new framework, or if potentially eco-genetic models (or other existing frameworks) could be used with some adaptations. If the conclusion is that existing frameworks might work equally well, then the manuscript could be shortened in this regard and perhaps end in another way, e.g. further ideas and suggestion for systems and questions that would be interesting to study.

My overall feeling however, is that the manuscript has a big point in highlighting that changes in which resource type is limiting might is likely to define eco-evolutionary responses to species loss or other perturbations. More precisely I think that most theory on evolutionary responses to environmental change in ecological communities mainly has considered adaptation to shifts in "substitutable" resources as opposed to "essential" resources. For example, quite some theory considers evolution in which species shift from one food resource to another, or shift from using one habitat to using another (substitutable resources). In contrast, a shift from food limitation to predator limitation represents imply a shift from one essential resource (food) being limiting to another essential resource (predator-free space) being limiting. Similarly, both nest holes and food are essential resources for many bird species. Shifts in which essential resource is limiting may have more profound effects than shifts in substitutable resources.

But if the manuscript would make an argument that shifts in limiting resources is a neglected theme in ecoevolutionary responses, that might need some support. Ground-breaking theory resource limitation in essential as opposed to substitutable resources by Tilman (e.g. 1980) could be cited. Some eco-evolutionary consequences (in terms of optimization and co-existence) of essential/substitutable resources are discussed by Vincent et al. (1996). A review by McGill et al (2006) which argues that community ecology has focused a bit too much on pair-wise interactions and distinct preferences (for substitutable resources) might also be relevant. A recent paper by Higginson (2017) discusses how nest sites in birds and pollinators is now becoming a more limited resource and leading to competitive exclusion in systems which were previously more structured around competition for food.

Resource limitation plays a role also in controlling the outcome of competition when resources are substitutable. For example, if two predators compete for two prey species, the predators which can supress the population abundances of the prey species to the lowest level and yet survive will win the competition. The predator can co-exist if they have different preferences however and each supress their preferred prey. This occurs in Lotka-Volterra-style food web models for example, and is fairly well studied. I suppose the manuscript is not about that, but perhaps this is worth clarifying? 


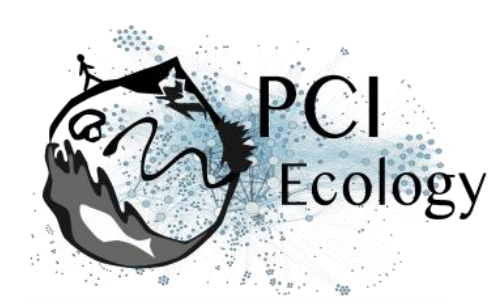

References:

Higginson, Andrew D. 2017. "Conflict over non-partitioned resources may explain between-species differences in declines: the anthropogenic competition hypothesis." Behavioral ecology and sociobiology 71.99 .

Tilman, D. (1980) Resources: a graphical-mechanistic approach to competition and predation. Am. Nat. 116, 362-393

Vincent, T. L. S., et al. 1996 Trade-offs and coexistence in consumer-resource models: it all depends on what and where you eat. The American Naturalist 148:1038-1058.

Mcgill, B. J., Enquist, B. J., Weiher, E., \& Westoby, M. (2006). Rebuilding community ecology from functional traits. TRENDS in Ecology and Evolution, 21: 178-185.

\section{MORE SPECIFIC POINTS}

Following up my points above I will provide some specific suggestions below regarding literature which I think the manuscript should be related to and which is some cases may require some larger adjustments of the text. The manuscript already has a lot of references, which is natural since it covers so many areas. I do not suggest all the below references needs to be cited. Rather, I hope that these suggestions would help defining the novelty in the manuscript a bit more and make it possible to shorten sections where previous work has been done.

\section{Eco-genetic modeling and individual based eco-evolutionary models}

The so called "eco-genetic" model framework presented by Dunlop et al (2009) bears many similarities with the framework proposed here (Fig. 5). Similar to here that model describes processes at many scales. Specific building blocks includes: evolving traits, inheritance model, individual growth model, density dependence, environmental variation, phenotypic plasticity, sex structure, mating systems and more. I have not done a detailed comparison, but it seems to me that the proposed framework in principle corresponds to such an eco-genetic model. Potentially the genotype-phenotype mapping is not included in the Dunlop et al framework, but on the other hand that framework includes a nice take on plasticity (via reaction norms) which seems generic and not discussed here. In Dunlop et al there are further references to applications of eco-genetic models (in fisheries). These studies give some idea of the strengths and potential weaknesses of this approach.

A recent review of individual-based eco-evolutionary models of different complexity is further provided by Romero-Mujalli et al. 2019.

References:

Dunlop ES, Heino M \& Dieckmann U (2009). Eco-genetic modeling of contemporary life-history evolution. Ecological Applications 19: 1815-1834

Romero-Mujalli, Daniel et al. 2018 Individual-based modeling of eco-evolutionary dynamics: state of the art and future directions. Regional Environmental Change 1:1-12.

\section{Evolution in species interactions}

The manuscript discusses evolution in species interactions (e.g. last para on page 22 to top of page 24 and page 30). There are relatively few citations here in spite of this being a huge research area, especially within the field of adaptive dynamics, and addresses many issues including community evolution, speciation and diversification (see refs below). I think this should be acknowledged, however I also think it might be possible to argue that there has been little focus on resource limitations and essential resources here (I think, but I am not $100 \%)$. Another reason to link the manuscript more to the field of adaptive dynamics and related 
frameworks is that the manuscript once published then could become more easily accessed to a broader modelling community, which I think will be inspired by the ideas in this manuscript.

Some references:

Loeuille, N. and Loreau, M. 2005. Evolutionary emergence of size-structured food webs. Proc. Natl. Acad. Sci. USA, 102: 5761-5766.

Dieckmann U \& Doebeli M (1999). On the origin of species by sympatric speciation. Nature 400: 354-357

Van Dooren, TJM, M Durinx and I Demon 2004 Sexual dimorphism or evolutionary branching? Evolutionary Ecology Research 6: 857-871.

Abrams P. A. 2001. Modelling the adaptive dynamics of traits involved in inter-and intraspecific interactions: An assessment of three methods. Ecology Letters 4:166-175.

Ripa J., L. Storlind, P. Lundberg \& J. S. Brown. 2009. Niche co-evolution in consumer-resource dynamics. Evol. Ecol. Res. 11: 305-323.

Brännström $\AA$ et al. 2012. Modeling the ecology and evolution of communities: A review of past achievements, current efforts, and future promises. Evolutionary Ecology Research 14: 601-625

\section{Theory regarding ecological and evolutionary consequences of species removal}

The ecological consequences of species removal from an interactive community has been studied quite a lot. The classic study by Paine (1966) could be cited as it identifies keystone species, i.e. species which if removed have a large impact on the community structure, relevant for present manuscript. Theoretical studies have tried to identify keystone species in model food webs. One interesting phenomena which may occur when removing species from a community is "community closure", i.e. once you have removed some species they cannot reinvade again, i.e. the community has changed irreversibly (Lundberg et al. 2000). That in turn links to the idea of "alternative stable states" and "attraction domains" in ecological systems where removal of species can lead to irreversible changes and trophic cascades (i.e. when a significant perturbation or removal of important (keystone) species moves the system from one domain of attraction to another).

Johansson \& Dieckmann (2009) introduces the Evolutionary Domain of Attraction (EDA) as an evolutionary analogue to the ecological concept. The idea there is that if you perturb an evolutionarily stable community (an ESS community), for example by temporarily subject it to a new selection regime, ensuing evolutionary responses may or may not restore the original community after the perturbation. Some ESS communities may have a large EDA meaning that it will return to the original ESS also after large perturbations. In some cases, a system may have multiple possible evolutionary equilibria (several locally stable ESS solutions). In such systems a perturbation may cause a transition from one ESS to another. The guppy system discussed in this manuscript could be thought of as a system with two evolutionarily stable states. The addition/removal of predators causes the evolutionary transition from one ESS to another. It is conceivable that the removal of a guppy prey species instead would not cause such drastic changes. Perhaps the lost prey species may over time be replaced by a similar one, by speciation or invasion and the original system be restored. Such a perturbation would hence not cause a transition to another ESS: the community would stay within the evolutionary domain of attraction of the original ESS community and be restored after the perturbation.

There is also a connection between the guppy discussion and the concept of "evolutionary keystones" introduced by Brown and Vincent (1992). In their model, removal of the predator caused evolutionary convergence in the niche positions of their prey, resulting in competitive exclusions. Hence the presence of the predator was key to maintain coexistence among the prey. Similarly, Johansson \& Dieckmann studied the removal predator species from a slightly more complex food web of 5 species including two predators. After removal of one of the predators, the original system is restored, but when removing the other triggers evolutionary responses which collapses the food web. In this system there are thus two alternative 


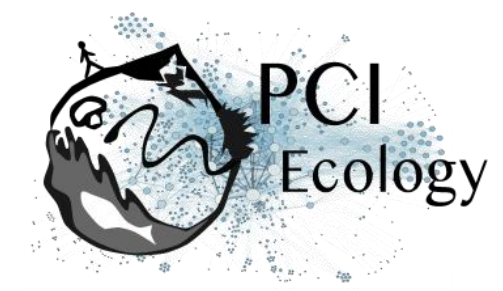

evolutionary stable states. An evolutionary keystone species can thus be seen as one which if removed causes the system to enter another evolutionary domain of attraction.

For the section of trophic cascades caused by evolutionary change, it might be relevant to cite theoretical work dealing with the issue of evolutionary change in one species causing severe changes in population densities of other species. One model studied by Bronstein et al (2004) considers co-evolutionary extinction cascades in mutualistic networks. Another theoretical study (Georgelin et al 2015) considers plant-pollinatorherbivore communities and shows that evolutionary changes in herbivores triggered by environmental change (pesticide use) may cause extinctions among pollinator species.

These studies are generally based on more minimalistic models than the framework proposed in the present manuscript. Many of them consist of Lotka-Volterra population dynamic models where the interaction coefficients are trait-dependent and selection gradients derived directly from the population dynamic models. Therefore, they cannot make predictions regarding for example population structure. They nevertheless show that many aspects of eco-evolutionary responses to species removal can be studied using relatively simple approaches.

\section{References}

Bronstein, Judith L., Ulf Dieckmann, and Régis Ferrière. "Coevolutionary dynamics and the conservation of mutualisms." (2004).

Brown J. S. \& T. L. Vincent. 1992. Organization of predator-prey communities as an evolutionary game. Evolution 46:1269-1283

Georgelin, E et al 2015 Eco-Evolutionary Dynamics of Plant-Insect Communities Facing Disturbances: Implications for Community Maintenance and Agricultural Management. Advances in Ecological Research. 52: 91-114.

Lundberg, Per, E. Ranta, and V. Kaitala. 2000. Species loss leads to community closure." Ecology Letters 3 : 465-468.

Johansson, J., \& Dieckmann, U. (2009). Evolutionary responses of communities to extinctions. Evolutionary Ecology Research, 561-588.

Paine, R. 1966. Food web complexity and species diversity. Am. Nat. 100: 65-75.

Solé, R.V., Montoya, J.M. and Erwin, D.H. 2002. Recovery after mass extinction: evolutionary assembly in large-scale biosphere dynamics. Philos. T. Roy. Soc. B. 357: 697-707.

DETAILS:

Page 4, middle

"Obviously, when $a=0, E($ lambdat $)=V E . "$

This is not really obvious from the approximation $a=\log (\operatorname{lambdat})-V E$. If one linearizes $\log (\operatorname{lambdat})$ one gets log(lambdat) approximately equal to lambda_t -1 . Perhaps I am missing something.

Page 12

"More generally, in such cases some heritable phenotypes will have long-run stochastic population growth rates that are greater than 0 , others will necessarily have rates that are less than zero, but the average longrun stochastic growth rates across competing phenotypes will be $0 . "$

Here you could cite Ripa \& Dieckman 2013 who considers evolution in stochastic environments (both for clonal and sexual (diploid) heritance) Ripa, J., \& Dieckmann, U. (2013). Mutant invasions and adaptive dynamics in variable environments. Evolution, 67(5), 1279-1290. http://doi.org/10.1111/evo.12046

Page 14 


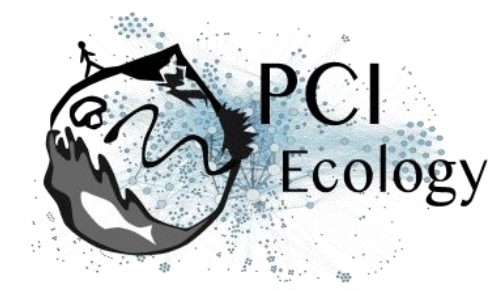

"This is most easily achieved by assuming that competing strategies are clonally inherited with (near) perfect fidelity (Metz et al. 1995). For sexually reproducing species this assumption is violated."

With sexually reproducing species it becomes trickier to study evolution of competing strategies. But the adaptive dynamics approach (i.e. Metz et al. 1995) has been extended to sexually reproducing species and can thus be used in this context:

Metz J. A. J. \& C. G. F. de Kovel. 2013. The canonical equation of adaptive dynamics for Mendelian diploids and haplo-diploids. Interface Focus 3: 20130025.

See also Ripa \& Dieckmann above.

Page 27

"A third route to large body size is the ability to access resources that may be unavailable to smaller individuals."

Perhaps trees fit in here as well? Evolutionary arms race to access light and suppress competitors. I am just curious.

Page 30

" Such a process will occur in systems where resources are limiting, where density- and frequency-dependent selection operates, or where coevolution is observed (Roughgarden 1971, Thompson 1999). "

Here is a good place to cite adaptive dynamics papers mentioned above, because most of them considers frequency and density dependent selection.

Fig. 1.

It would be good with worded titles of the top panels, just like in the bottom panels and the mathematical symbol in the top panel looks odd. What $=1.046$ ? Perhaps also colour the population dynamics in B with red to get a consistent coloring scheme which can be immediately appreciated from the figure.

Figure 3.

Here I think it would be good with some more details about the simulations (for reproducibility). It seems like the original distribution of traits (the $z_{-} i \mathrm{i}$ ) are drawn from a normal distribution. How are the offspring generated?

Signed by Jacob Johansson

Reviewed by anonymous reviewer, 2019-02-03 21:18

Review on 'CAUSES OF DEATH AND FAILURES TO REPRODUCE, LIMITING RESOURCES, ECOLOGICAL DYNAMICS, AND SELECTION: HOW TO EVOLVE A LOW PREDATION GUPPY, AND CAUSE A TROPHIC CASCADE' by Coulson T

This manuscript describes the case how structural models can aid in understanding ecological population dynamics and natural selection - particularly Integrative Population Models and Individual Based models. The manuscripts first describes well in detail the components of life-history-fitness linkages and how these may be implemented in a modelling framework. In particular, the author discusses the role of various sources of 'resource limitation' in driving fitness and selection. For most parts the manuscript is clear and pleasant read and would be a valuable contribution.

However, the sections (starting page 31) on how to implement these models, left me wanting. I would like to see a discussion how we can better integrate empirical data collection with implementing such models - as it seems that some of the short comings of our ability to use these in natural populations come from lack of 
right kind of temporal population size, demographic and life-history/trait data, and data on the key limiting resources in any given system. Currently it would seem to be possible to apply such models to a limited number of model systems. I think the field would progress more if we could aim to collect more of relevant long-term data in nature (in addition perhaps to implementing similar data on more controlled but manipulative systems on organisms with sufficiently fast generation times). Would be great if the 'data needed' aspect was explicitly covered and the non-modeller readers among us would be advised on what type of data would be useful to collect for increasing inferential power and rigour of such structured models - so that they could be implemented on a wider range of natural systems.

Sometimes the use of references is insufficient, and the manuscript is rather long and could be shortened somewhat - I make some suggestion below to this end. I hope my comments help to improve it further, as I think it would be a useful paper for many evolutionary ecologists.

Specific comments that I hope help to increase readability \& value further:

P2: I found the start of the introduction, using the empirical guppy example a bit lengthy - before coming to the main goal of the manuscript. It is nice to illustrate with an empirical case the biological relevance, but I think the first 2 paragraphs could be condensed to essential. Particularly since the guppy example is repeatedly returned to in different places. In fact, it might work best if the guppy system, to the parts relevant to the topic at hand, was described in a separate box - to which one could refer to in the text. (The first 3 lines on page 3 "The guppies....factors that limit the population's growth" could in fact be moved earlier on, for a sharper start)

P5: As a non-modeller I had to check the word 'moments' used in this context. Might be useful (if also empiricists are targeted) to clarify such jargon.

P5: I found the reasoning for the use of clonal versus sexually reproducing species in different aspects a bit confusing. May be useful to explicitly state why in some place clonal reproduction and in other sexual reproduction is assumed (= why is not one or the other used for the different section - or more interestingly both compared).

P6-1st line P7: I would like to see clear mentioning of the caveats of making inferences about historical determinants of selection - else this statement seems rather trivial, at least for within species comparisons.

P7, last 3 lines: The relevance of comparing the scenario of two different equilibria, with both $a=0$, is not clear. Perhaps provide empirical example to illustrate this. Neither is it clear why the shift between the two equilibria is expected to last only a few generations. Is there a basis for this?

P13, 2nd paragraph. This paragraph seems to me to be linked to traits that allow adjustments to buffer against environmental variation = phenotypic plasticity in physiology, behaviour, morphology etc. Does this refer to phenotypic plasticity only or are there other forms of traits that allow resilience in face of environmental variation? Or does it not matter for how selection operates whether the 'resilience increasing' traits are plastic?

P14, 1st paragraph: I would imagine that in several empirical study systems, it would be possible to compete the different life-history strategies against each other empirically also. Would be useful to state (for those who work on such malleable systems) how this could be empirically directly tested (also to confirm results of simulations). Also, state more explicitly why this assumption is violated for sexually reproducing species and what difference it makes. Can we only test clonally reproducing species? If so, how strong are our inferences?

P14, 2nd paragraph: you mean variation in developmental plasticity / ontogeny? Would be useful to clarify and exemplify.

P15: I think this is a bit thin argument for what we can do (and should do) to build better genotypephenotype-fitness maps, such as could be achieved by investing G-P maps in more detail and via high throughput phenotyping (e.g. Houle et al. 2010 review on Phenomics) and accounting for gene-phenotype network structure. 
P17, 3rd paragraph, line 3: this would seem to me to assume that the food source productivity (e.g. grass or algal production) is stable and does not evolve in response (which in many cases of biotic interactions of herbivory or predator-prey does not hold). How does the scenario change in eco-evolutionary feedbacks where the food source may evolve? Same holds for $p$ 18, 2nd paragraph: failure could also be if assumed that the food does not evolve in response to consumption?

P18-19. I found the heading of 'Inheritance - genetic and otherwise' intriguing but the content somewhat disappointingly not covering the recent discussions on non-genetic inheritance. How this matters for our inferences on evolution, for example via cross-generational effects of resource limitation, would be a useful addition. Right now the content covers standard population genetic/quantitative genetic (Va) based inferences. P19: earlier in the text evolution was defined as either allele frequency change or heritable phenotypic change. Here only allele frequency changes are covered primarily and the linkages with different inferences based on heritable phenotypic change could be better covered.

P21, 1st paragraph. The forms of non-genetic inheritance is rather poorly covered here. It would be useful to more explicitly state the main types, as well as how (if) it affects our inferences about ecological and evolutionary processes. The recent book by Bonduriansky and Day on "Extended heredity" could be a useful reference here. And perhaps at least briefly touch on under the structural models section how these could be implemented and what information is needed for us to be able to infer the relevant contribution of different modes of inheritance on direction and magnitude of eco-evo feedbacks.

P22: how does the within species variance (rather than mean distance) affect our inferences and predictions on co-evolution? Also P23: The concept of individual specialization and how it links to eco-evolutionary feedbacks could be better covered here.

P26: I could not quite follow from all that was written earlier, why we now focus on body size - comes abit out of the blue- Of course body size is a key life-history trait and typically a strong fitness determinant, as well as intimately linked to resource mediated selection (via metabolic requirements) - but this could be made more explicit to make clear why body size receives this extended treatment in the manuscript. References for metabolic theory of ecology should be better covered in here.

P27, 2nd paragraph: the island rule comes out of the blue and relevance is not clear for the general goal of the manuscript. Seems to take away attention from the core. In general, I find the body size evolution section in need of streamlining and condensing. It just seems to bring different alternatives for body size evolution and some seem rather peripheral - yet is not explicit enough how this will help us with those structural models... and evolution of life-histories / eco-evo feedbacks in general. Would be good to streamline this and link better to the goal of the manuscript.

P30: In general, unless we are talking of population dynamics (of one focal species or of two interacting species) I am not sure we expect the dynamics to continue 'ad infinitum'. I think such continuous process takes place under certain assumptions (the same factors feeding back on each other, the continued ability of the target species to evolve etc...).

P30: 2nd paragraph, line 5: It would be perhaps useful to consider the potential for indirect eco-evolutionary feedbacks - that may be much harder to both track. Also, how predictable would we expect such feedbacks to be (i.e. when the ecological selective agent and the phenotype determining fitness may not influence each other directly)?

P31, 2nd paragraph: I am convinced that we would require significant amounts of data - much more, and much more detailed, than is available for most empirical systems. However, not stating how we could overcome this challenge, seems a bit unsatisfactory. Also, that we are not able to measure eco-evo feedbacks in many systems with currently available data, does not mean that they do not occur in nature. Hence the last statement could be modified to something 'Although it is empirically difficult to demonstrate ecoevolutionary dynamics in nature, in some cases eco-evo feedbacks have the potential to generate pronounced eco-evo dynamics'. I would like to see a clear definition for eco-evo feedbacks versus eco-evo dynamics, references to empirical work that has been able to show such consequences, as well as 
suggestions (if possible to make) under which situations we expect eco-evo feedbacks to lead to dynamics. (I think that for the field to advance, we should be more consistent in separating feedbacks and dynamics, although other seems to put all under the umbrella of dynamics.)

P31, 'Tying strands together'.

- I would have liked to see a lead here to the complexity of the real world (see also Hendry 2019 'Critique of eco-evolutionary dynamics', Functional Ecology Special Issue), the type of data needed to do so, and how making sense/tracking the dynamics can be aided by understanding the processes and models laid out in this manuscript.

- The importance of mating system could be made more explicit and be an interesting part of the discussion in context of eco-evo dynamics

P33: I found the set-up of the paragraphs for the different functions somewhat confusing - yet these components (functions of survival, reproduction, development, inheritance) are really important for predictions of eco-evolutionary feedbacks as well as understanding the models. Would be good to structure the text for clarity (perhaps also numbering the functions by 1,2, 3, 4 would be helpful for the reader, see minor comments below).

As to function 3 (development) - this seems to me reflecting plasticity (including developmental plasticity) of the phenotype. This could be more explicitly stated. In general, I think we need more attention to this component in understanding eco-evolutionary feedbacks (e.g. given that the plastic components of the phenotype can be an important determinant of speed and magnitude of ecological change, and these can change over the course of the ontogeny or life-time of the organisms).

P34. To me the treatment of the inheritance function is rather narrow. Most standard approaches to eco-evo feedbacks only consider additive genetic effects, whereas non-genetic inheritance, or genetically determined parental (typically maternal) effects can strongly affect evolutionary speed and direction - and on the same token, we would expect also effects on eco-evo feedbacks. Any detailed treatment to this end is not needed for the current manuscript, but I think it would be useful to make the point explicit that these other forms of cross-generational effects may alter the scenarios based on additive genetic inheritance.

P35-36: 2nd paragraph. As noted in my general comment above, it would be useful to state what we (empiricists) need to do to be able to use these models - more explicitly state the type of data needed. Which ecologically relevant eco-evo model systems are possibly suitable for this? I find the long list of different models conducted a bit too abstract to be useful - it does illustrate the many different aspects, but perhaps would work better as an overview table? Can we say something more about when each type of model is best used or how they can be integrated - to create that 'single model' that captures better the organization of the different components influencing eco-evo processes? Do we not need the data that allows building the details of these models?

P38, 1st paragraph. Saying that such models are 'frequently not analytically tractable' begs the question of what should we do then? Can we overcome this? Or what do we do with the models at all if they are not tractable?? Perhaps the last paragraph - using models that do not have to capture all feedbacks, but still can be informative about core processes, is meant to be one solution. Which is fine, but could be better stated.

P39. 'What can we say without models' section was rather uninformative. It basically seems to present what empiricists can do by hard work and conducting a lot of well-designed field studies and experimental manipulations. What I would like to see is how we best can take the power of both worlds, integrating the models with empirical work to inform each approach of the best way to tackle the core questions at hand (e.g. which life-stage is the most important in mediating eco-evo feedbacks, how does sexual selection influence eco-evo feedbacks in contrasting ecological environments, which species interactions in a foodweb are likely to result in eco-evo-dynamics, how does the mode of inheritance influence direction an magnitude of eco-evo feedbacks, etc etc). 


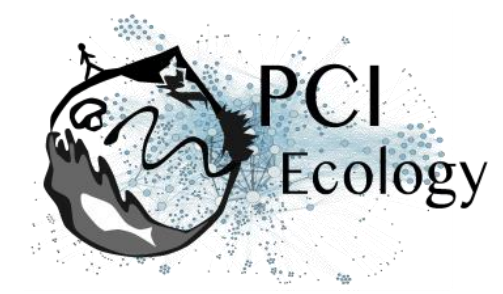

Page 39

- the case of the guppy. Is the most interesting question to be addressed really 'why low predation environments result in parallel evolution of phenotypes'? Wouldn't the simple answer be there is parallel divergent selection (loss of predators'? Wouldn't it be more interesting to understand HOW this parallel selection operates (the eco-to-evo pathway in the feedbacks) and how do these parallel phenotypic changes influence eco-evo feedbacks ? Again, some of this text is rather repetitive and adding a box with the guppy system as an example case would help making just the case of relevant points without the need to repeat the text in other places of the manuscript.

- Although the emu case (I assume the start was not an e-mail chick, Page 39, 2nd paragraph... ..) is somewhat entertaining as a heuristic thought exercise, I did not find the 1.5 page description necessary nor informative for our understanding.

Minor (RWD= reword):

At several places it seems original work is not well referenced (statements made without reference) - I indicate those below.

P6, 1st paragraph, 5th line - RWD to 'increasing survivorship or fertility at any age...'

P6, line 6: provide the reference for the case of Elk in Yellowstone

P7, line 4: State explicitly that this refers to guppies experiencing high predation environments. (Note that this is an example case were it would work perhaps better to have the guppy system presented in a separate box). Same unclarity holds for P8, 2nd paragraph (guppy example). This refers to the case of guppies inhabiting low predation environments?

P7, line 5: RWD to 'Many of the phenotypic...'

P7, line 19. This statement about 'prior to removal of predators' is confusing. Does this refer to an empirical case study with experimental removal?

P9, Line 2: allow who to survive? Check wording of this sentence for clarity.

P9, 1st paragraph, last 3 lines. It would be helpful for the naïve empirist to have a reference for selection differentials and need to understand patterns of inheritance already here (I am not that naïve reader, but I think this may be useful for others that may not be familiar with evolutionary inferences - but still may work on relevant empirical study systems).

P9, 3rd paragraph, line 7: RWD to ...' result in selection on phenotypic traits associated with detection, ....'

P9, 3rd paragraph, line 9: I think we can not assume that any population 'will' adapt - without making further assumption about trait heritability and lack of evolutionary constraints. RWD to something like 'Given sufficient time, and that assumption underlying evolutionary responses (e.g. that traits are heritable), the population may adapt and express adaptive traits ...' (Else sounds rather deterministic).

P11, line 4: clarify that this means the mean fitness of the population.

P11, line 6 RWD to ' consequence of this is that the...'

P11, 2nd paragraph, line 6: RWD to ' non-zero selection differential...'

P11, last paragraph: This hole nesting bird sentence is unclear and confusing. RWD.

P12. State at first mention what the beta's refer to (beta0 and beta1).

P12, lines 1-4: I found these sentences unclear. Why do we expect this and how is this evolutionary suicide manifested in this case ? L 3: RWD to '..., evolution will favour fewer, larger individuals...'. Again saying that 'evolution will result' sounds too deterministic. 


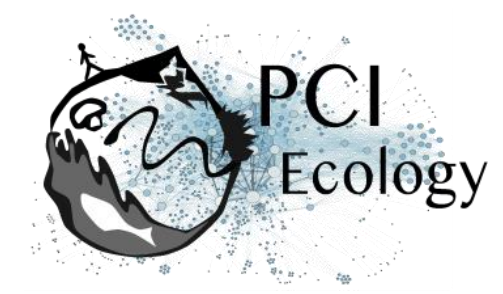

P13: It was not clear to me what the first alternative of evolution was. RWD perhaps to 'I now consider how evolution can proceed by reducing VE'.

P13, last paragraph: Not clear why ploidy of species matters. State more explicitly.

P16, 2nd paragraph: State for the non-expert reader what the breeding value is - or at least provide reference.

P16, 2nd paragraph, line 4-5: RWD the last sentence of gene expression and how environment can affect gene expression. (e.g. what are the 'environmental drivers? Also sentence structure unclear). In general, this section against seems to relate to phenotypic plasticity (via gene expression) yet this link is poorly made.

P17, 2nd paragraph, line 1: you mean developmentally plastic traits - or traits that are expressed at maturity or that are impacted by senescence ? RWD.

P17, 2nd paragraph, line 10: RWD to '...they will have large values...'

P18, 3rd paragraph, line 4: RWD to ' ... is that there is little competition...'

P18, 3rd paragraph, line 2: provide references for these statements about how artificial selection operates, as well as for line 7 on quantitative genetic covariances, and for evidence for these methods working well in absence of the covariances.

P20, 3rd paragraph line 2: RWD to ' base pair substitutions...', line 3: RWD to insertions..' line 6: unclear what is meant by 'such' genes. Clarify. Line 8: provide reference for this insight on mutations

P20. It would be useful to be more specific here as to which definition of epigenetic inheritance is referred to here (the narrow definition of methylation etc alteration or wider parental effects).

P21, 2nd paragraph: It would be useful for those not accustomed to think of eco-evo feedbacks to explicitly state that an important difference these biotic resources make (as opposed to non-biotic) is that they can evolve in return.

P21, last paragraph. Provide reference for interaction coefficients capturing functional responses and conversion rates.

P22, 2nd paragraph: 'However' seems redundant here. Remove.

P24: It would be useful to have the subheading $f$ 'Trophic cascades' here. Line 4, provide reference and definition or empirical example of trophic cascades. Last 2 lines on this page could be moved after the 1 st full paragraph for easier reading.

P25, 2nd paragraph. This is to me generally a very unclear paragraph. For instance, does it mean evolution of any other species altered the dominant causes (and hence selection) of death and successful reproduction in a dominant species - or rather the evolution of the dominant species itself - or either ? RWD for clarity. RWD to 'In such a case, the dominant species was unable...'. Also, what is meant by dominant species? Dominant in numbers? Dominant in role in ecosystem (aka keystone species)?

P25, 3rd paragraph: Again I think it would be easier to make these arguments more streamlined if the guppy case would be overviewed in a separate box.

P26, 2nd paragraph - 3rd line: Provide reference for 'relative metabolic rate' - 4th line: RWD to ...'than those that are smaller ' - Why is it key that the exponent is less than unity - for the current discussion?

P27, 2nd paragraph - line 6: RWD to 'For example, food-limited populations of...'

P29, Eco-Evolutionary feedbacks 


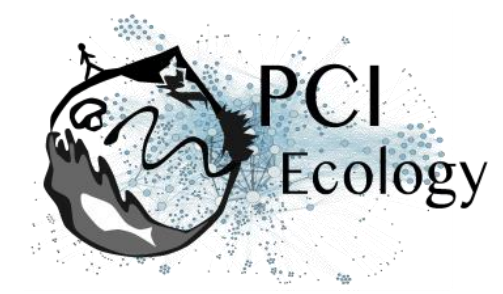

- Is the reason that compelling empirical evidence is missing for eco-evo feedbacks that they are poorly defined or that they are difficult to demonstrate? I would rather think the reason is the latter (though I also agree that they are often poorly defined).

- Here again the contrast in definitions earlier on in the manuscript for evolution defined as change in allele frequencies or heritable phenotypic change. The allele frequency change is the narrowest sense, but given the increased realization that non-genetic (at least non-DNA sequence change) inheritance mechanisms appear common, I would consider these other alternatives. Especially since for ecological relevance of the eco-evo feedbacks any transgenerational effects can be important.

- Line 4: RWD to '...is frequently defined as the dynamics of populations, communities,...'. Or otherwise give a clearer definition (rather than how they are measured). Also: provide reference for the definitions. Line 5-6 'If we stick with this definition...' seems redundant, delete.

- Line 8: I think we need the mediating effect of the phenotype for allele frequency change to have any eco-evo feedbacks.

- 2nd paragraph, line 2: you mean constant positive trait-fitness association ? I guess we would not expect exponential growth of the population if the association was negative.

- 2nd paragraph - It would be easier to follow this argumentation (biological relevance) if here the reader was reminded of what the denominator and numerator of the selection differential equation represents.

P30, 1st paragraph: The sentence on line 2-3 ('What all this means...') seems repetitive to what as said in the previous paragraph.

P30, 3rd paragraph. What type of 'parameters' do the beta's present? Slope of relationships? Any?

P31, 1st paragraph, line 4. RWD to 'increasingly'

P32. Would be useful to have a subheading 'Modelling eco-evolutionary feedbacks' -before going into the models.

P32, 3rd paragraph, line 5: I don't understand what this really means '...the number of individuals within a population with each combination of components of the phenotype' ? Be more explicit.

If these models are to be generally usable, perhaps refer to statistical packages that are available for users (if there are such)?

P33, line 3: which two functions? Which other functions? This becomes clear below but these can be tied together and made easier to read if 'two functions (i.e. survival and reproduction) and two other (i.e. development and inheritance)...')

P38, 1st paragraph, linen10: The sentence of '...have spurred on the modelling approaches I have been involved in developing' is not very informative and can be deleted. The manuscript is lengthy as it is.

P40, last paragraph, line 8: RWD to '...which phenotypic trait will evolve ' (or which phenotype will evolve?)

P41, 2nd paragraph. Provide references for presumed predation pressure on ground feeding birds. Is the idea that emus lost the ability to fly prior to predation becoming a significant source of mortality based on phylogenetic or historical inferences or some such (in which case references would be appropriate) or only speculation ? In general, I find this emu section rather speculative and also uninformative. In particular the last paragraph could be completely be left out.

Last sentence of conclusion. I do think that the next step would be conduct studies in different populations where the limiting factor differs - or has changed recently so predictions can be made and eco-evolutionary (or ecological and evolutionary) dynamics observed. But it seems to me we generally require more data and, in particular, be able to identify the key limiting factor (which may require substantial data in most system) to then test whether the framework proposed in this manuscript helps us to make more accurate predictions. 


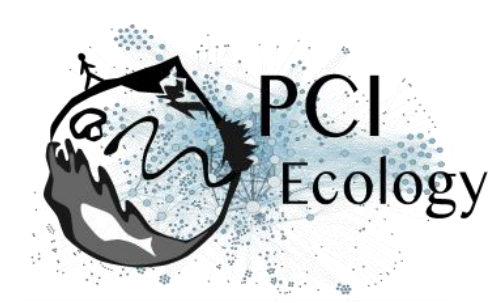

\section{Author's reply:}

The DOI link takes you to the revised ms.

Download author's reply (PDF file) 